Real-time monitoring of a positron beam using a microchannel plate in single-particle mode

To cite this article: G. Vinelli et al 2020 JINST 15 P11030

View the article online for updates and enhancements.

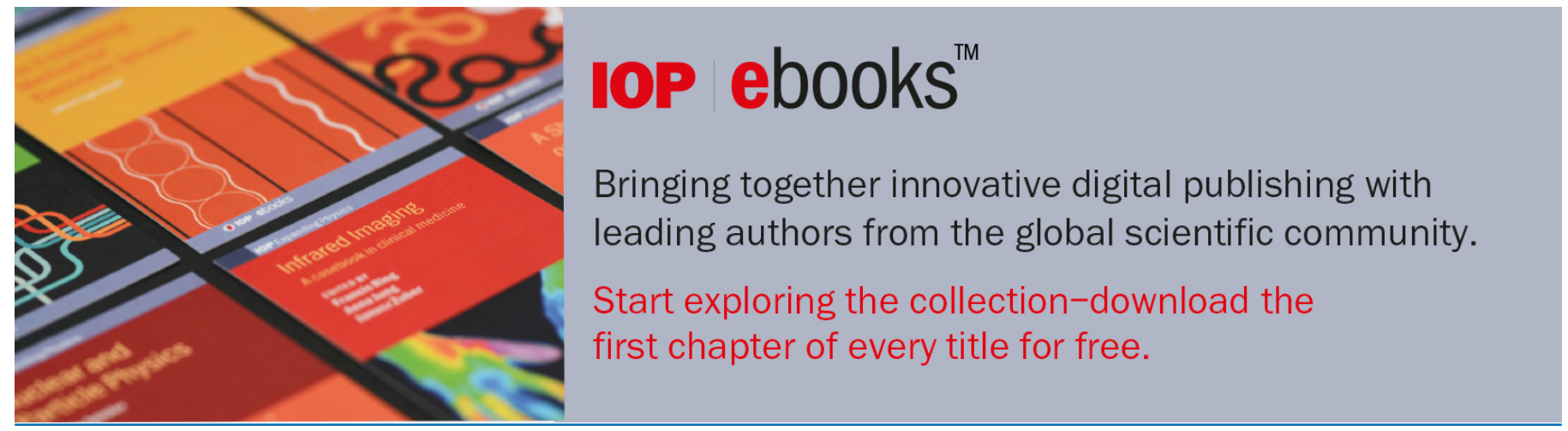




\title{
Real-time monitoring of a positron beam using a microchannel plate in single-particle mode
}

\author{
G. Vinelli, ${ }^{a, b}$ R. Ferragut, ${ }^{a, b}$ M. Giammarchi, ${ }^{b}$ G. Maero, ${ }^{b, c}$ M. Romé ${ }^{b, c}$ and V. Toso ${ }^{a, b, 1}$ \\ ${ }^{a}$ L-NESS and Department of Physics, Politecnico di Milano, \\ Via Anzani 42, 22100 Como, Italy \\ ${ }^{b}$ INFN, Sezione di Milano, \\ Via Celoria 16, 20133 Milano, Italy \\ cDepartment of Physics "Aldo Pontremoli”, Università degli Studi di Milano, \\ Via Celoria 16, 20133 Milano, Italy \\ E-mail: valerio.toso@polimi.it
}

ABSTRACT: This work presents a real-time diagnostic method to monitor and characterize a positron beam by using a microchannel plate (MCP), a phosphor screen and a CCD camera. We propose a digitizing method based on single-particle detection. This approach offers the possibility to quantify the particles per unit time that interact with the MCP. This type of detector is based on a consolidated technology and its efficiency has been demonstrated for nuclear radiation and particles in many experiments. This work also presents the detection efficiency as a function of the positron kinetic energy in the range from $50 \mathrm{eV}$ to $17 \mathrm{keV}$, essential for a complete characterization of the particle beam. The method allows the characterization of a positron beam in real time with a spatial resolution of the order of tens of microns.

Keywords: Accelerator modelling and simulations (multi-particle dynamics; single-particle dynamics); Beam-line instrumentation (beam position and profile monitors; beam-intensity monitors; bunch length monitors); Particle detectors; Real-time monitoring

\footnotetext{
${ }^{1}$ Corresponding author.
} 


\section{Contents}

1 Introduction 1

2 Materials and methods $\quad 2$

3 Digitization and beam spot characterization method 4

4 Study and simulation of the overlapping effect $\quad 8$

5 Efficiency as a function of the positron energy 10

$\begin{array}{lll}6 & \text { Conclusions } & 14\end{array}$

\section{Introduction}

Microchannel plate (MCP) detectors have been frequently used for particle detection to measure Xrays, ultraviolet rays, neutral particles as well as charged particles such as electrons, antiprotons, etc. (see refs. [1-7] and the papers cited therein) due to their high quantum efficiency, and the relatively high temporal and spatial resolution. Even if the spatial resolution is limited in comparison with other types of detectors such as nuclear emulsions [8-10], MCPs offer advantages, for example the possibility to monitor a particle beam in real time. In many cases, this is also possible thanks to the development of new digital techniques. Another limitation of the MCPs is the difficulty of discriminating the incident energy and the type of particle that interacts with them. These limitations can be dealt by using complementary techniques such as mass spectrometry and timeof-flight measurement.

During the last decade, imaging systems based on the use of a phosphor screen as an anode for the MCP have gained considerable popularity. The reason is that this method provides a position measurement from single particles to a large number of particles, thus making the design of a 2D imaging system relatively simple. For single particles, the detection usually involves a stack of at least two MCPs.

In this work we have characterized a system consisting of an MCP, a phosphor screen and a CCD camera with a continuous positron beam. The beam is located at the L-NESS (Laboratory for Nanostructure Epitaxy and Spintronics on Silicon) in Como (Italy), and has a fixed implantation energy in the range between $50 \mathrm{eV}$ and $17 \mathrm{keV}$. The spatial resolution and the absolute efficiency of this detector have been measured as a function of the positron kinetic energy. The typical decay time of the phosphor light $\left(\tau_{10 \%}=0.2 \mu\right.$ s for a P46 phosphor type and $\tau_{10 \%}=1.5 \mathrm{~ms}$ for a P43 type), coupled with the ability to operate with a short exposure time of the CCD camera (milliseconds or less), allows the identification of each single particle interacting with the MCP. By acquiring and digitizing a large number of frames, it is also possible to monitor the particle beam in real time: events 
are individually digitized and summed up in order to obtain a higher interaction event statistics. By analyzing via software the set of events thus acquired it is possible to obtain relevant informations on the beam, such as its spatial position, shape, width and intensity. This characterization could be complemented with an accurate high-resolution arrival time detection $[4,11]$. A tool with such a rapid response can be very useful not only in the preparation of an experiment, for example to center and focus the beam, but also as a real-time detector with trigger functions. This methodology is applicable to either continuous or pulsed beams, the latter by synchronizing the particle bunch with the CCD.

One of the motivations of this work is, in light of the present results and technological improvements, to evaluate the utilization of MCPs in the field of antimatter interferometry and deflectometry. Recently, positron waves and antiproton projection patterns were distinguished by means of high resolution nuclear emulsions [10, 12]. The advantages of a real-time detector could mean a breakthrough in this field of research.

The paper is organized as follows: section 2 presents details of the MCP and, in general, of the experimental setup. Section 3 presents the digitization method proposed to identify and quantify individual particles from the spots acquired by the CCD camera. This section also illustrates that both the individual particle spots and the positron beam distribution recorded acquiring a significant number of particles follow in good approximation a Gaussian profile. The spatial resolution of individual particles and the characterization of the beam profile using the MCP are presented. Based on simulation results the overlap effect of the single spots is discussed in section 4 . Section 5 presents an experimental estimate of the detection efficiency, essential for quantifying the intensity of the positron beam.

\section{Materials and methods}

The microchannel plate (MCP) detector used in this work is produced by Hamamatsu Photonics (Model F2223-21P) and consists of a 2-stage chevron (v-like) stack of microchannel plates made of lead glass coated with a nickel-based alloy (Inconel). The effective size of the MCP is $27 \mathrm{~mm}$ in diameter, while channels have a diameter of $12 \mu \mathrm{m}$; they are tilted at an $8^{\circ}$ angle with respect to the longitudinal axis of the MCP. The open area ratio of this MCP is $60 \%$. By applying a positive voltage difference between the entrance and the exit of the channels (set in the present study at $+1.4 \mathrm{kV}$ ), an interacting particle produces an electron shower via secondary emission. Each individual microchannel acts as a continuous dynode electron multiplier, amplifying the input signal with a gain in the $10^{5}-10^{7}$ range, depending on the supply voltage [13]. For this study the MCP is coupled to a P43 type phosphor screen with an effective size of $24 \mathrm{~mm}$ in diameter, set at a distance of $1 \mathrm{~mm}$ downstream of the $\mathrm{MCP}\left(\mathrm{L}_{2}\right.$ in figure 1). The size of the phosphor grains is of $2-3 \mu \mathrm{m}$. This phosphor features a peak emission wavelength of $550 \mathrm{~nm}$ and a decay time of $1.5 \mathrm{~ms}$ at $10 \%$ of the light intensity [14]. Thanks to a potential of a few $\mathrm{kV}$ (set to $+3.9 \mathrm{kV}$ in this work) between the exit of the MCP and the phosphor, the electron shower is accelerated to hit the phosphor grains. Here, thanks to the fluorescence process, the electron avalanche is converted into photons, which can be observed by means of a CCD camera. The light intensity detected by the CCD camera is proportional to the number of electrons. In addition, it should be taken into account that the detected light intensity depends on the decay time of the phosphor grains and the exposure 
time of each frame, which in this work is typically $50 \mathrm{~ms}$. The measurements are performed in a condition of very low dark current, i.e. producing $<0.05$ events by frame in the MCP area.

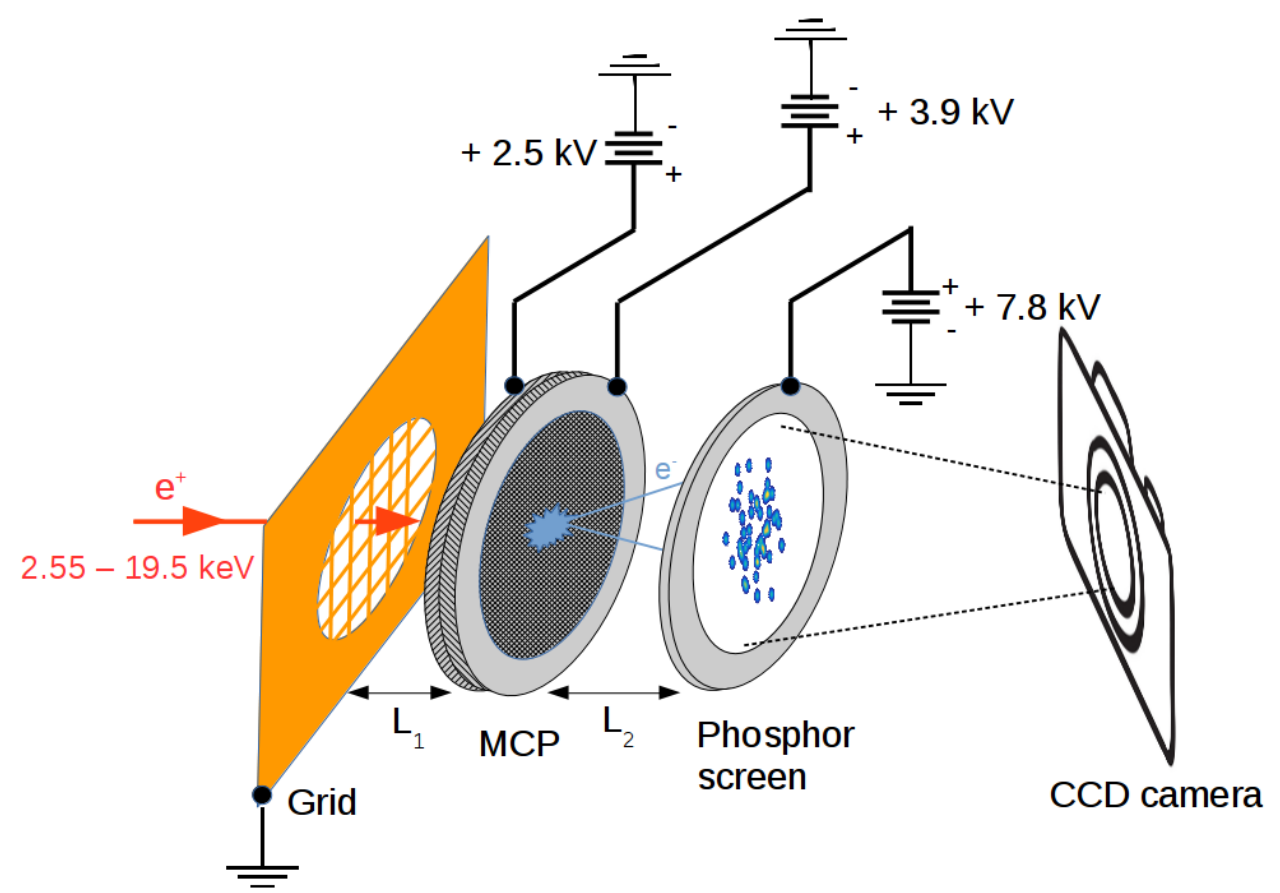

Figure 1. Schematic representation of the experimental setup with the positron deceleration module.

This system $(\mathrm{MCP}+$ phosphor screen $+\mathrm{CCD})$ has been tested with positrons in the energy range $50 \mathrm{eV}-17 \mathrm{keV}$, with the same positron beam used in the QUPLAS (QUantum interferometry and gravitation with Positrons and LASers) experiment $[10,15]$. Positrons are emitted by a ${ }^{22} \mathrm{Na}$ source that undergoes $\beta^{+}$decay. A fraction of the emitted positrons is moderated into a mono-crystalline tungsten foil (W[100], $1 \mu \mathrm{m}$ thick), and thanks to their negative work function in tungsten, is emitted at low energy (about $2-3 \mathrm{eV}[16]$ ). Once emitted, positrons are accelerated (up to reaching a maximum energy of $19.5 \mathrm{keV}$ ) by means of a purely electrostatic and continuous system and guided to hit the MCP. The beam, the MCP and the phosphor screen operate in vacuum at pressures between $10^{-6}$ and $10^{-8} \mathrm{mbar}$, while the digital camera is in air. A glass window provides the optical connection between the camera and the phosphor. The camera lens is used to focus the phosphor region onto the CCD sensor and to adjust the amount of collected light (by varying the diaphragm aperture). The beam width is a few $\mathrm{mm}$, depending on the energy, about 2-2.5 $\mathrm{mm}$ for incident energies $\geqslant 2 \mathrm{keV}$ and a little wider at lower energies (between 2.5 and $3.5 \mathrm{~mm}$ ).

To carry out the measurements of the MCP efficiency as a function of the kinetic energy of the positron beam described in section 5, a suitable configuration is chosen to ensure that the positron intensity at the MCP is independent of the incident energy of the particles. A sketch of this apparatus in shown in figure 1. For this measurement, to prevent losses in the transport of low energy positrons, the minimum kinetic energy used for the beam is $2.55 \mathrm{keV}$. The grid is grounded and the first face of the MCP is connected to a positive potential $(+2.5 \mathrm{kV})$. For all measurements positron are decelerated just before the MCP (a distance $L_{1}=5 \mathrm{~mm}$, see figure 1). For example, 
positrons reaching at the maximum energy, $19.5 \mathrm{keV}$, to the grounded grid, then hit the MCP with a kinetic energy of $17 \mathrm{keV}$. The same $2.5 \mathrm{keV}$ gap by electrostatic deceleration occurs for all incident energies. Therefore, the positron kinetic energy range at the MCP surface varies between $50 \mathrm{eV}$ and $17 \mathrm{keV}$. The grid is formed by wires with a diameter of $30 \mu \mathrm{m}$ and a pitch of $1.2 \mathrm{~mm}$, giving a $95 \%$ transmission of the particles.

\section{Digitization and beam spot characterization method}

Positrons coming from the beam interact with the MCP according to the processes described in the previous section and appear on the phosphor screen as bright spots in an image that is digitized using a CCD camera.

If for a given exposure time the image contains more than one spot, it is not possible to exclude the possibility of overlapping. The overlap probability depends on several factors, as we will see later in section 4: the number of the single spots, their size, etc. When it is necessary, for instance, to characterize a continuous beam and, in particular, quantify the beam intensity, a statistically significant number of particles is required (e.g. $10^{4}-10^{6}$ particles). In this case, it is important to avoid or at least to minimize the overlaps. Overlapping often makes it impossible to distinguish different signal sources, thereby reducing detection efficiency, i.e. the observed number of spots for a given frame may be less than the actual number of positrons. There are some algorithms for estimating signal overlap, even for Gaussian distributions [17, 18]. In this work we have studied how to minimize this effect and thus avoid, whenever possible, the use of these algorithms. One strategy is to acquire many short-lived frames and merge them later via software. The necessary total exposure time depends on the beam intensity and on the spot size. Taking into account the characteristics of the present setup, we have typically set $50 \mathrm{~ms}$ as the frame exposure time. Figure 2a shows an example of a frame (from now on we will call "frame" the digital image taken for a given exposure time, $50 \mathrm{~ms}$ in this case). Each frame can be seen as a matrix of pixels taking a value between 0 and 16,383 $\left(2^{14}-1\right)$ on a scale of light intensity (that is, 14-bit resolution of our CCD camera). The value 0 corresponds to a pixel that has received neither light signal nor noise, while the value 16,383 corresponds to a pixel that has reached full saturation. An example of the digital histogram matrix of a single particle is shown in figure 3. As will be discussed below, this histogram follows a nearly 2-D Gaussian distribution.

By means of software analysis, it is possible to estimate the mean background level (BL) of each frame image and its standard deviation $\sigma$. The criterion used here considers as signal everything that exceeds a threshold $T h=B L+3 \sigma$. An algorithm identifies the coordinates $(x, y)$ in the matrix corresponding to the centroid of each spot above the threshold. Figure $2 b$ represents the digitized positions of all spot centroids of the image shown in figure 2a, which is obviously independent of the arbitrary intensity of the spots. Recently, an alternative analysis technique of phosphor screen images was implemented, based on a convolutional neural network, with the aim of estimating the MCP gain [19]. This technique reduces the background noise enough to distinguish single-particle events, yielding results similar to those presented here.

Since the spots have a 2-D Gaussian intensity profile (figure 3) it is possible to perform a fit with a Gaussian function plus the previously estimated background. The free parameters of the fit are the centroid, the width, the amplitude and the rotation angle (when the Gaussian profile is 


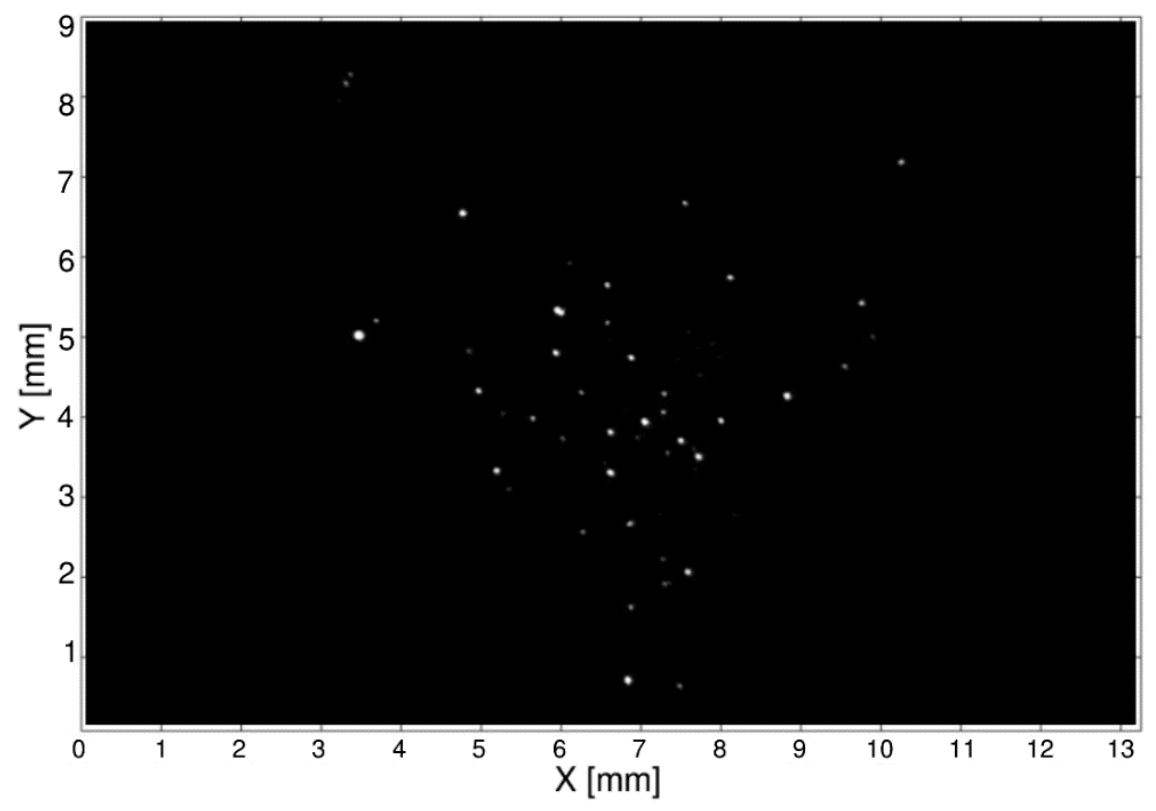

(a)

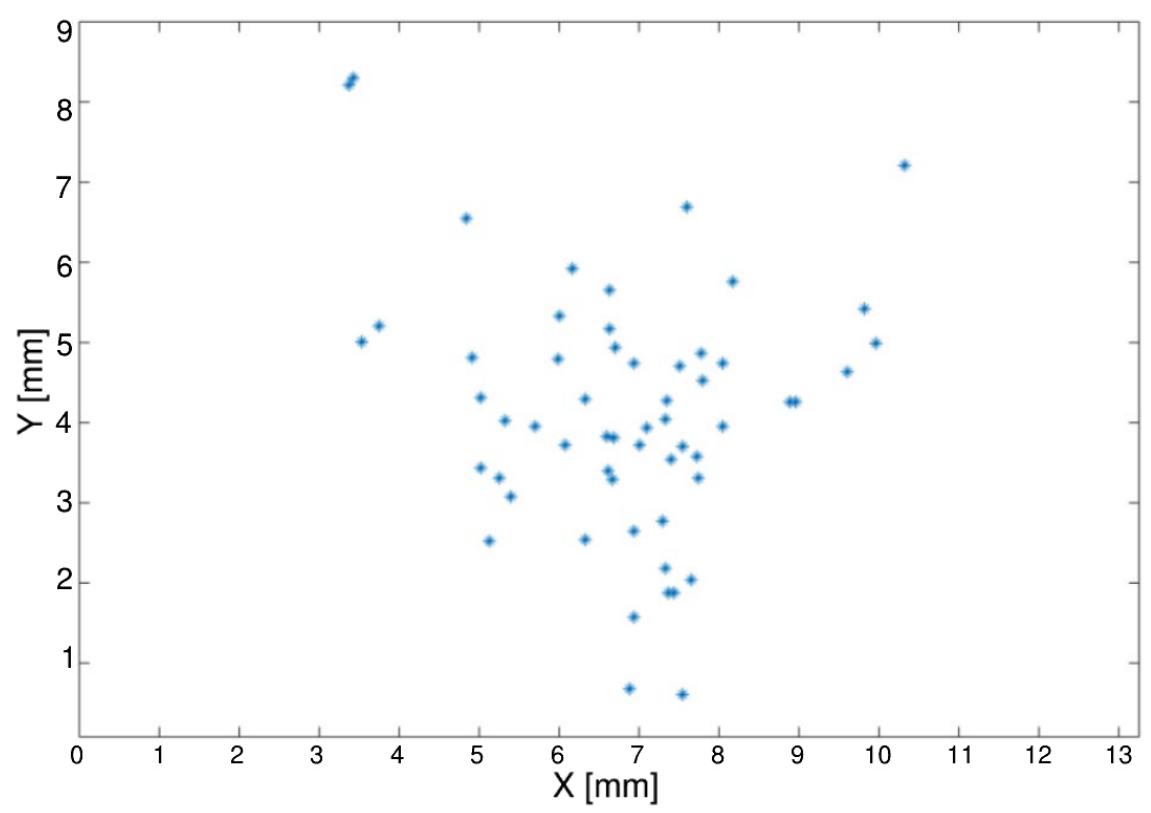

(b)

Figure 2. (a): Digitized image of the phosphor region acquired with the CCD camera. The white spots are the phosphor grains hit by the electron shower produced by individual positrons. The exposure time is $50 \mathrm{~ms}$. (b): Image representing the position of the centroid of each spot by means of blue crosses in a matrix corresponding to the image observed in (a). 
asymmetric). The software automatically performs the fit on all the spots in the frame. Figure 4 shows the Gaussian distributions contained in a frame. The standard deviations of the spots obtained by a fitting procedure provide useful information to estimate the spatial resolution of the acquisition system. In this case the average measured resolution of the single spot is $60 \pm 2 \mu \mathrm{m}$ (fwhm: full width at half maximum, note that the abbreviation "fwhm" is written in lowercase when referring to the single spot). This value depends on the potential drop across the MCP, which is chosen in order to optimize the resolution and the amplification, and on some geometrical and intrinsic characteristics of the setup such as the MCP channel diameter, the distance $L_{2}$ (figure 1) and the phosphor grain size. The secondary electron shower that reaches the phosphor screen has two velocity components, in the longitudinal and transverse direction. The longitudinal component is due to the potential difference between the MCP and the phosphor screen $(3.9 \mathrm{kV})$. The transverse one corresponds to the energy with which the secondary emission from the tubes takes place, that is an energy distribution up to $100 \mathrm{eV}$ (when the signal decays to $1 \%$ of the number of electrons emitted) [20]. To a first approximation, the overall shower trajectory between the MCP and the phosphor is represented by a cone. The emission angle of electrons can therefore be calculated as $\theta=\arctan \left(v_{y} / v_{x}\right)=1.5^{\circ}$. Taking into account the distance $L_{2}=1 \mathrm{~mm}$ between the MCP and the phosphor screen, the electrons hit the phosphor within a circular region with a diameter of $50 \mu \mathrm{m}$. On the other hand, the indetermination of the position of the arrival point of each positron is given by the channel diameter $(12 \mu \mathrm{m})$. These simple estimates give an idea of the resolution limit of the MCP in use, which is close to the one measured here.

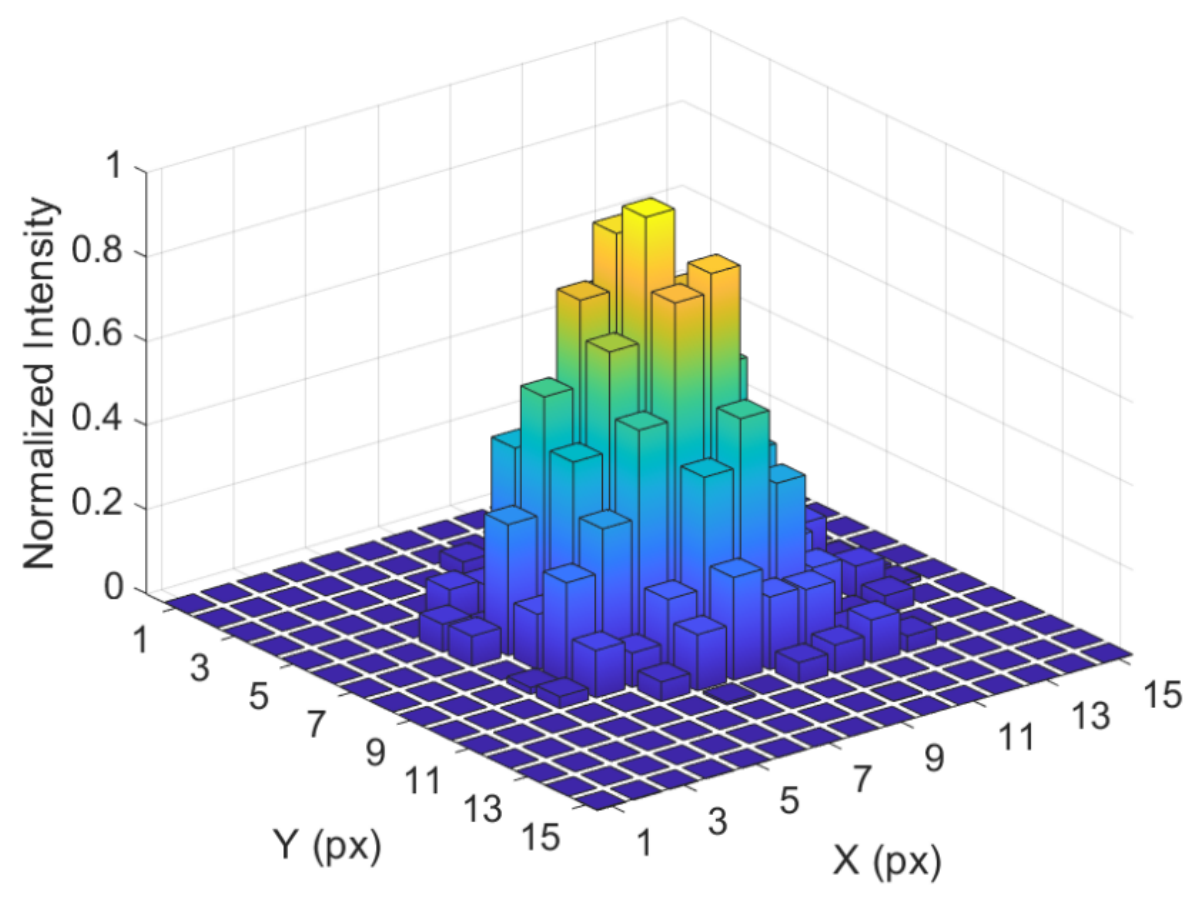

Figure 3. 3-D representation of the histograms acquired for a single spot in the digital matrix.

At the current state of the art it is possible to improve by an order of magnitude the spatial resolution measured in the present work with tens of picoseconds of time resolution [3, 4]. Therefore, the trend of technological improvements makes MCPs attractive for interferometry and deflectom- 


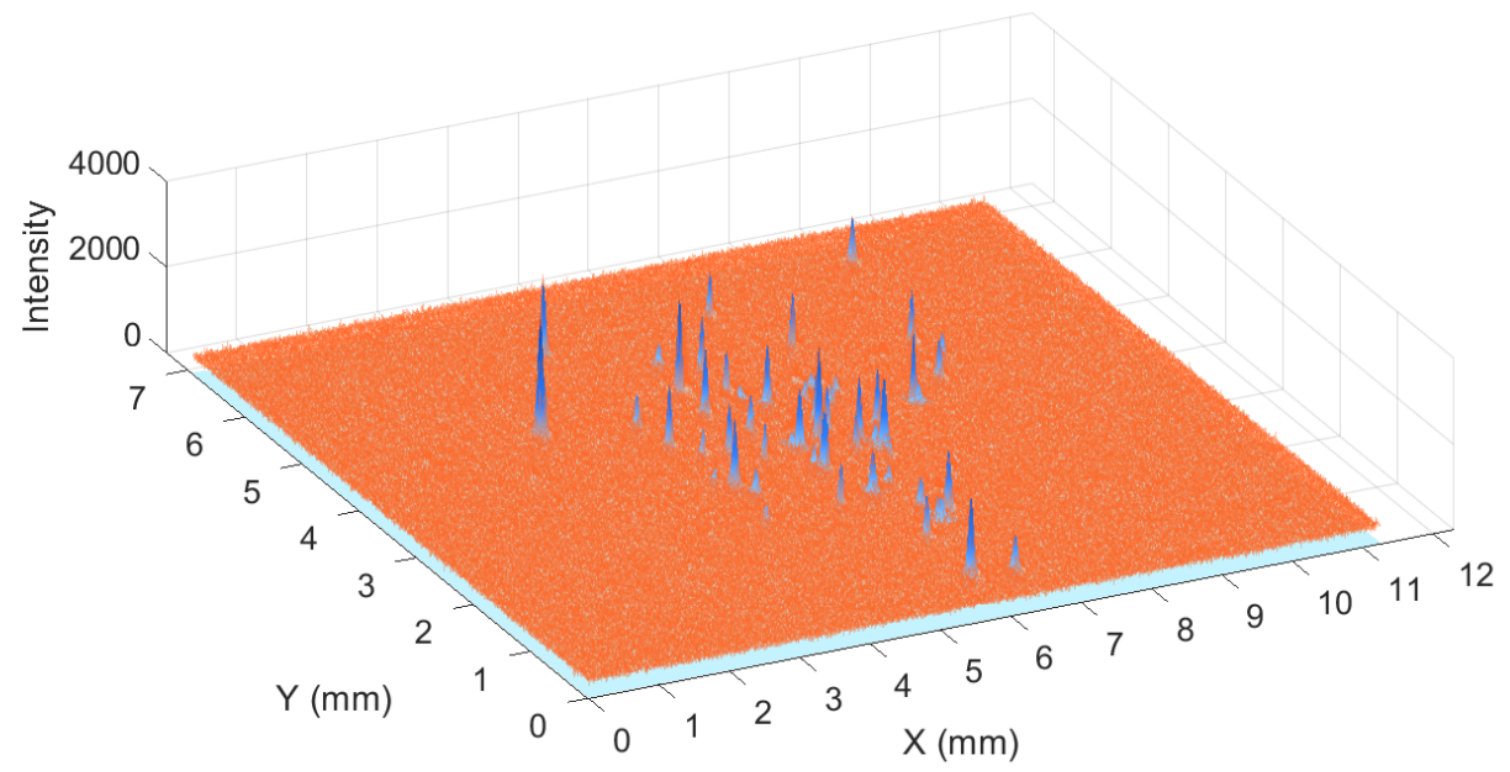

Figure 4. 2-D Gaussian fits of each spot for a given frame.

etry measurements of matter and antimatter particles or atoms (for instance, in the framework of the QUPLAS experiment) $[10,15]$. A strategy to use MCPs in antimatter interferometry to resolve nanometric or micrometric patterns is the utilization of magnetic magnification lenses as those used for the demonstration of single-electron double slit-like interference by Merli et al. [21] and then by Tonomura et al. [22]. Another strategy, recently used with an MCP to observe the hydrogen interferometry in the Talbot-Lau regime [23], consists in tilting at some angle ( $\sim 1 \mathrm{mrad})$ the third grating with respect to the two preceding gratings to magnify the pattern using the moiré effect [24].

As previously mentioned, it is useful to acquire camera frames with a limited number of spots in order to reduce the overlap probability. Hence it is possible to save the information obtained from the first image and proceed to the next frame. Each acquired data frame is converted to a digital matrix which has unit values in the pixels corresponding to positron arrival positions and zero elsewhere (like the example of figure $2 b$ ). The arrival positions are determined by finding the local maxima of the acquired data and using an algorithm to distinguish the real spots from the noise-generated ones. To do this, it is necessary to analyze the surroundings of each spot by dividing the matrix into squares having dimensions equal or greater than the resolution obtained experimentally ( $\geqslant 60 \mu \mathrm{m}$; $200 \mu \mathrm{m} \times 200 \mu \mathrm{m}$ in figure 5). This process is iterative and analyzes all the saved frames (four hundred, in this case). Once all the images have been analyzed, it is possible to superimpose all the matrices of each frame and thus recreate the overall beam intensity profile as shown in figure 5 . In this way, the statistical weight of each event is one instead of the arbitrary digitized scale given by the CCD camera. It is found that the beam profile distribution has a nearly Gaussian shape.

A Gaussian profile was recently observed in a previous work on the same apparatus using nuclear emulsions as a detector [25]. Performing a fit with a 2-D Gaussian function it is therefore possible to obtain useful information on the beam, such as position, width and symmetry. For instance, from the analysis of the distribution in figure 5 we obtain a number of spots of $\sim 2.6 \times 10^{4}$ in a detection time of 20 seconds ( 400 frames of $50 \mathrm{~ms}$ each, plus a dead time of about $100 \mathrm{~ms}$ 


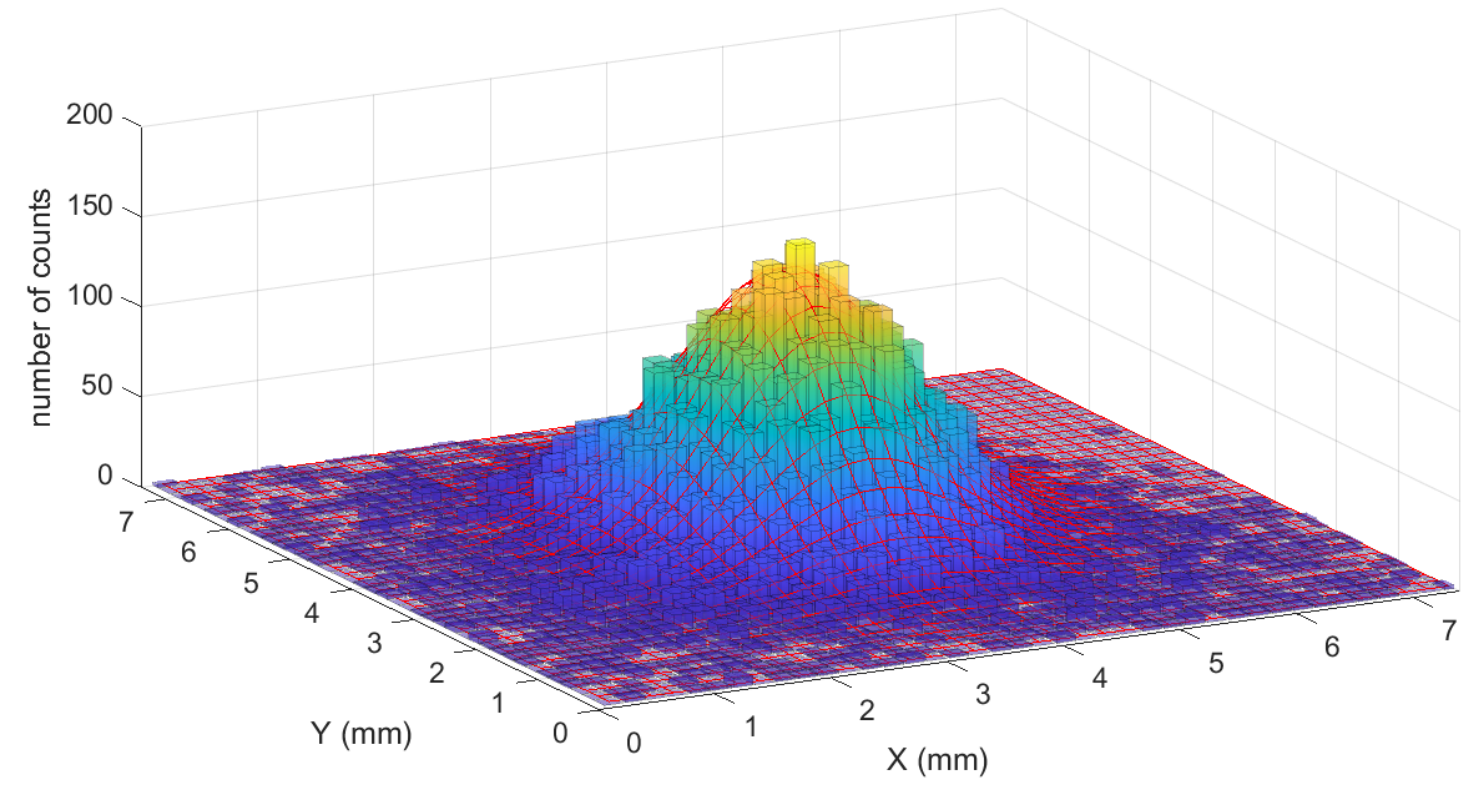

Figure 5. Overall beam intensity profile for monoenergetic positrons at $17 \mathrm{keV}$. The beam distribution is compared with the best-fit Gaussian function.

between frames) with $17 \mathrm{keV}$ monoenergetic positrons. This corresponds to a beam intensity of $(2.88 \pm 0.19) \times 10^{3}$ positrons/s (after calibration using the criteria to be presented in sections 4 and 5) and a mean FWHM (averaged over the $x$ and $y$ axes, note that the abbreviation "FWHM" is written in capital letter when referring to the overall spot) of $2.25 \pm 0.02 \mathrm{~mm}$. The chi-squared test ( $\chi^{2}$ divided by the degrees of freedom) of the Gaussian model approaches $1.5 \pm 0.2$. The last parameter typically lies within the range 1-2 depending on the statistics for a Gaussian distribution, the focalization and aberration corrections of the beam. Figure 6 shows a comparison of the radial average distribution of the intensities between the histogram and the Gaussian function of figure 5 . This comparison shows that the beam is well described by this function and is not affected by significant aberrations.

Another relevant information is related to the statistics of the individual spots that, with a certain efficiency (estimated in section 5), correspond to individual particles: in this case positrons that arrive at the MCP. In this way, it is possible to obtain a detailed geometric description and a good estimate of the real-time positron intensity of the beam. Only short acquisition and software processing times, seconds or a few minutes in total, are required, depending on the beam intensity and the required statistics.

\section{Study and simulation of the overlapping effect}

To better characterize the detection system, a numerical simulation has been developed. Its purpose is to reproduce the signal observed with the digital camera. This study helps to understand some effects, such as the overlap of the spots. The simulation requires some input parameters to faithfully reproduce the observations, which can be provided by the analysis of the acquired frames. These input parameters are: the number $N$ of spots and the distribution and size of the beam profile 


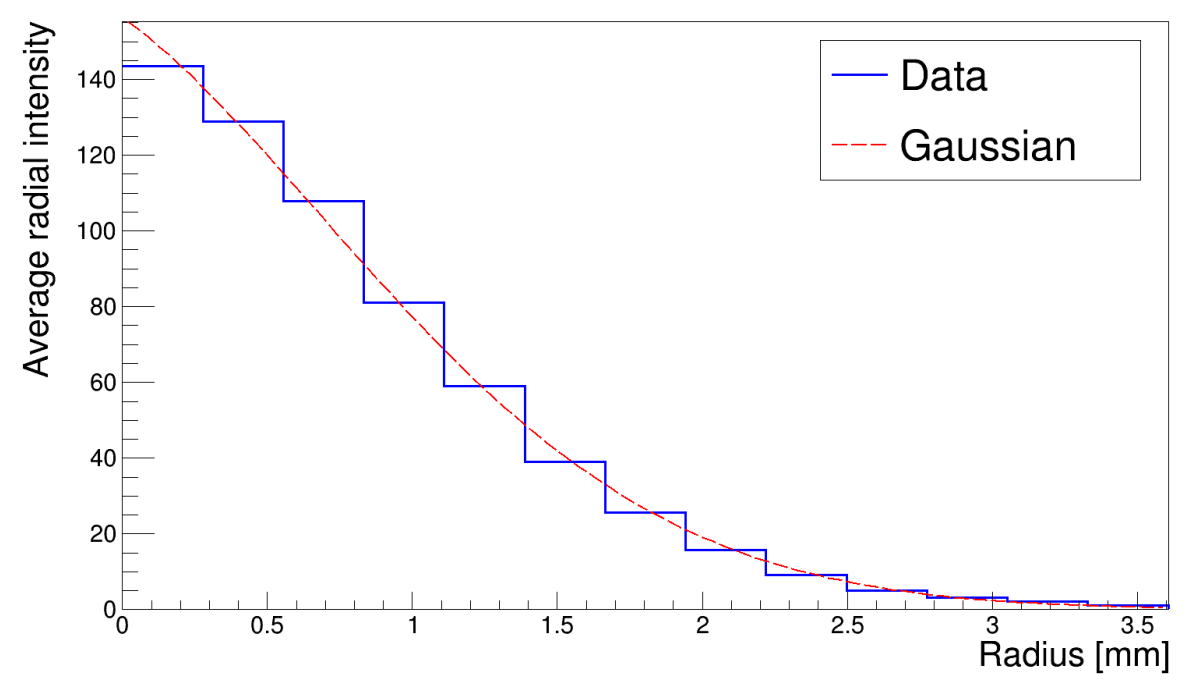

Figure 6. Radial average distributions of the intensities of the histogram data and the Gaussian function of figure 5 calculated starting from the position of its centers.

$f(x, y)$. To make the simulation even more accurate, the background has also been added in the form of a white noise reproducing the measured one.

The simulation randomly generates the centroid coordinates of the $N$ points, in agreement with their Gaussian distribution $f(x, y)$ observed in section 3. The spot size, according with measured data, is also randomly extracted for the $N$ points. An example is shown in figure 7 where the center of the black dots represents the centroid of the spots, while the horizontal and vertical bars represent their fwhm. The same procedure used for the experimental data analysis is reproduced for the simulated data in order to verify the method with high reliability.

To study the overlapping effect, it is necessary to define when two spots can be considered separated. We have considered two spots as separated when the distance between their centroids is larger than the sum of the respective half-widths (fwhm/2).

Therefore, by computing the distance between the center of the generated spots and knowing their width, it is possible to identify the overlapped ones (marked in red in figure 7). Given a number $\mathrm{N}$ of points, the simulation provides a number of overlapped spots. Since the extraction process is purely statistical, this number can however vary by repeating the simulation. By running the simulation many times (one thousand times in this case) it is possible to obtain an average value of overlapping spots. Considering the setup used for this work and the CCD integration time of $50 \mathrm{~ms}$, we obtain a probability of overlap $O v$ about $8.3 \%$ (e.g. data in figures 2 and 4 ).

The simulation can also be used to study how the overlap percentage is affected by some parameters, such as the number of spots or their width. Setting all parameters (in this case those obtained in section 3) except for the spot width $f$ of the single spot ( $f=\mathrm{fwhm}$, see for example figure 3), the overlap percentage varies as shown in figure 8a. In this plot, the x-axis represents the ratio $f / F$ between $f$ and the beam width $F$ (where $F=$ FWHM, average value of FWHMx and FWHMy of the overall beam, see an example in figure 5). This makes the overlap percentage independent of the overall beam size. As expected, the overlap probability approaches 0 when $f \ll F$, while it approaches saturation at $100 \%$ overlap when $f \geqslant 0.5 F$. 


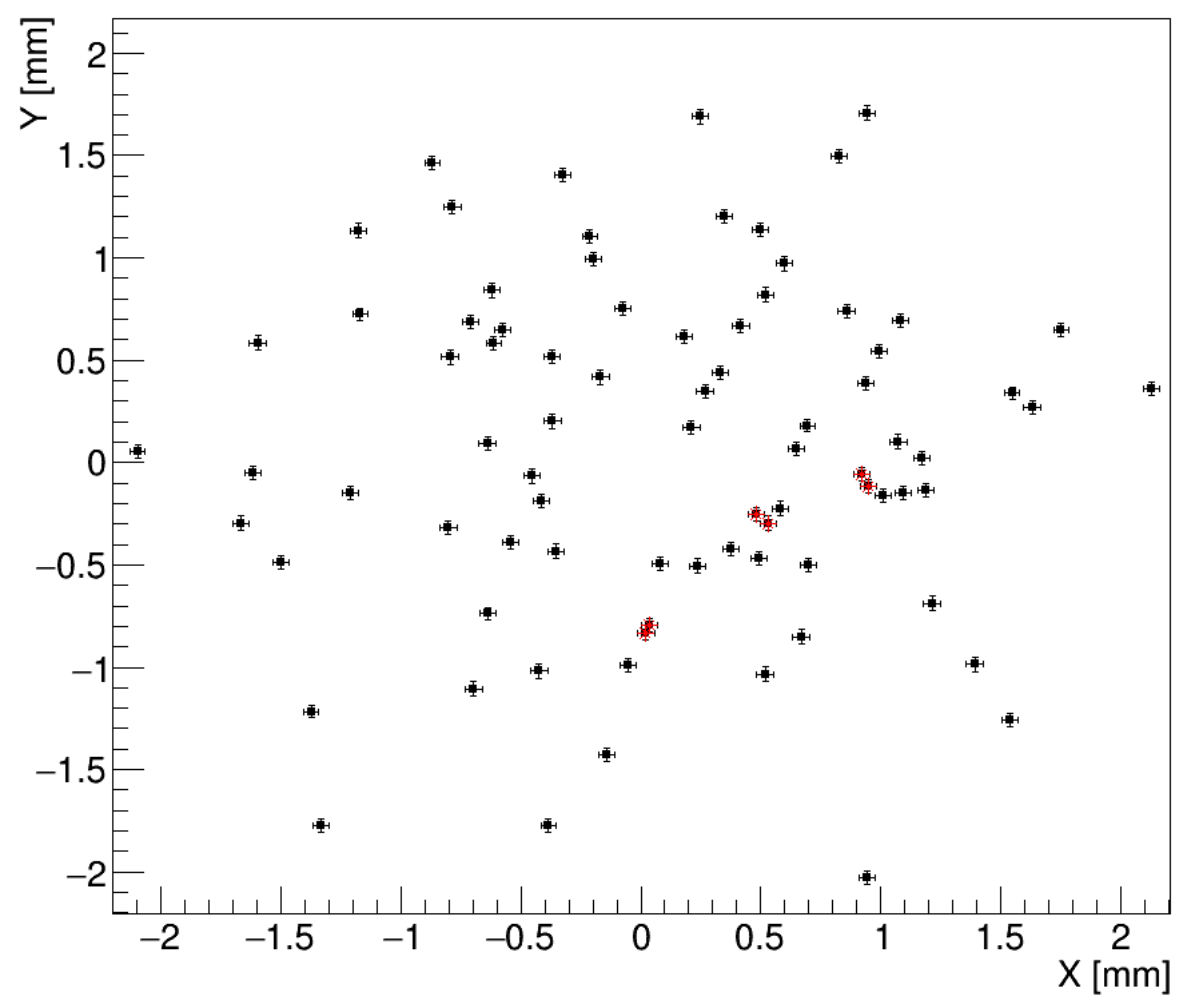

Figure 7. Simulation of the spots observed with the CCD camera. Analysis of simulated spots. The overlapping spots are marked in red.

Figure $8 \mathrm{~b}$ shows the overlap probability as a function of the number of spots, calculated with the same parameters obtained in section 3. Once again it approaches 0 when there are only few spots and saturates to a total overlap of spots when $\mathrm{N}$ reaches values approximately $>10^{4}$.

\section{Efficiency as a function of the positron energy}

As previously described, the software analysis identifies and characterizes the positron spots. By knowing the efficiency of the MCP, it is possible to integrate all frames to estimate the beam intensity from the total number of spots. The efficiency of an MCP depends on the particle energy.

Here we propose an indirect method to calibrate the beam intensity for positrons with a given kinetic energy and thus quantify the response of the MCP.

To estimate the positron beam intensity we use a calibrated ${ }^{22} \mathrm{Na}$ source encapsulated into the center of a volume of a Plexiglas (PMMA) support $\left(11 \times 27 \times 2 \mathrm{~mm}^{3}\right)$ and a high purity germanium (HPGe) detector. For this purpose, an identical replica of the PMMA of the calibrated source support was manufactured. The replica is punctured perpendicularly in the center of the area $A$ $\left(11 \times 27 \mathrm{~mm}^{2}\right)$ with a nearly conical hole (average diameter $\left.1.5 \mathrm{~mm}\right)$ up to the middle of the depth $(1 \mathrm{~mm})$ of the support. Positrons are then implanted in the direction perpendicular to $A$ in the hole center (see figure 9a).

The idea is to reproduce the emission position and attenuation of the annihilation gamma rays $(511 \mathrm{keV}$ ) of the calibrated source. The positron beam is implanted at high energy (about $3 \mu \mathrm{m}$ at 


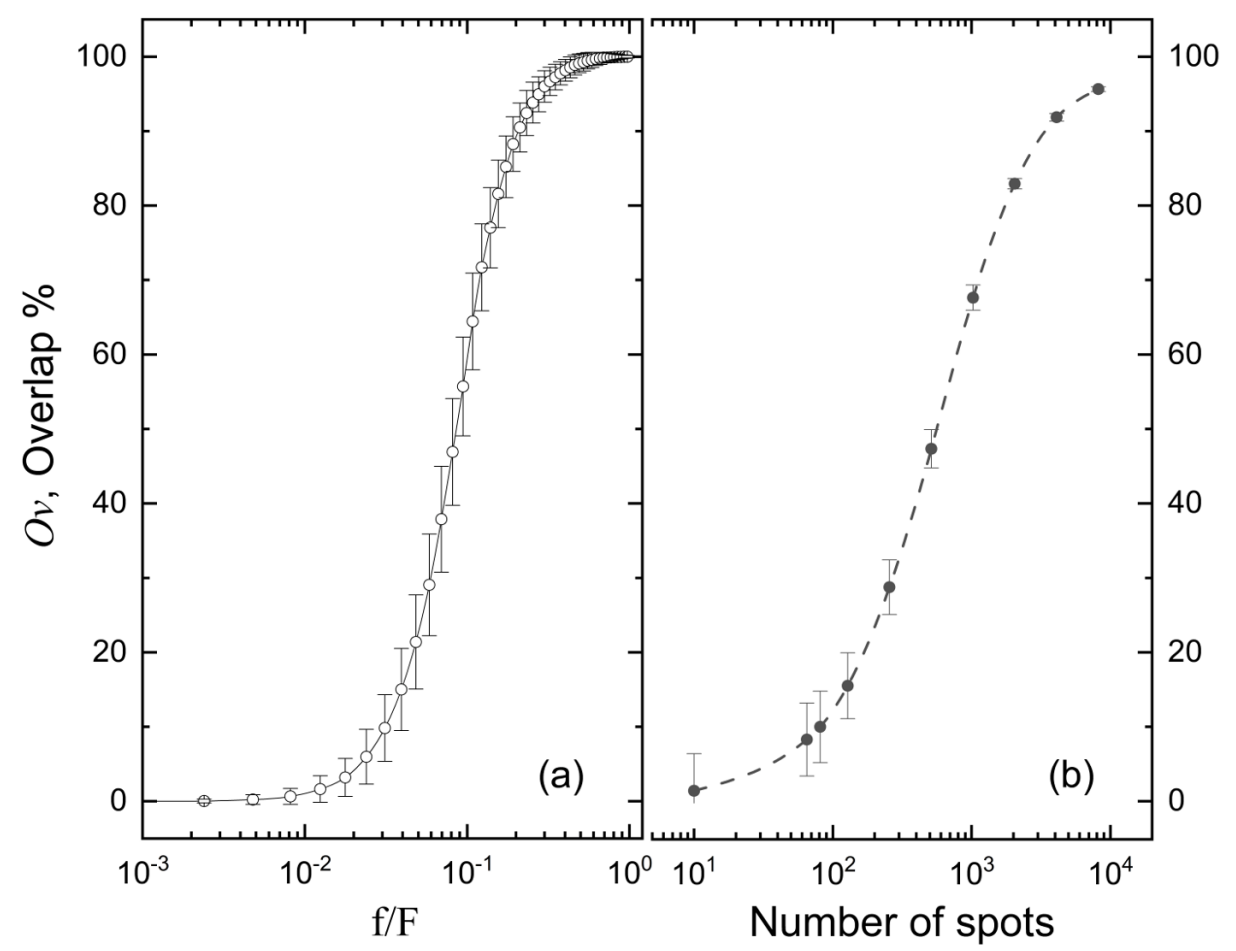

Figure 8. (a): Overlap probability as a function of the ratio $f / F$ between the spot width and the beam width (b): Overlap probability as a function of the number of spots.

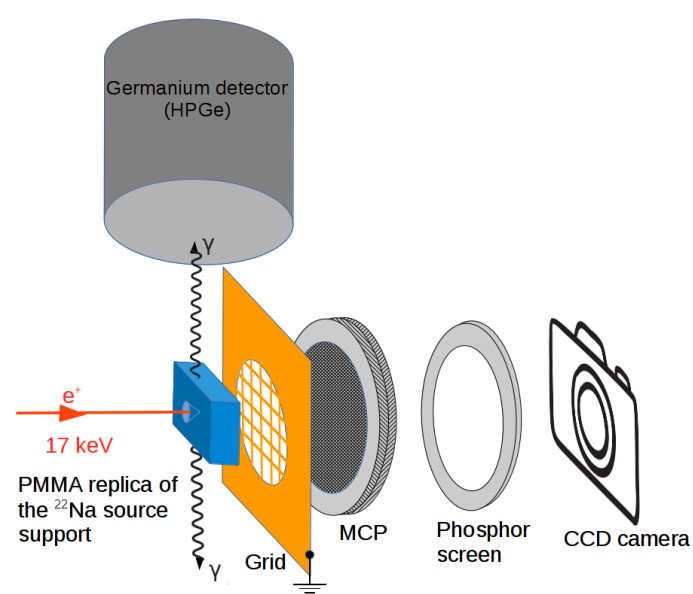

(a)

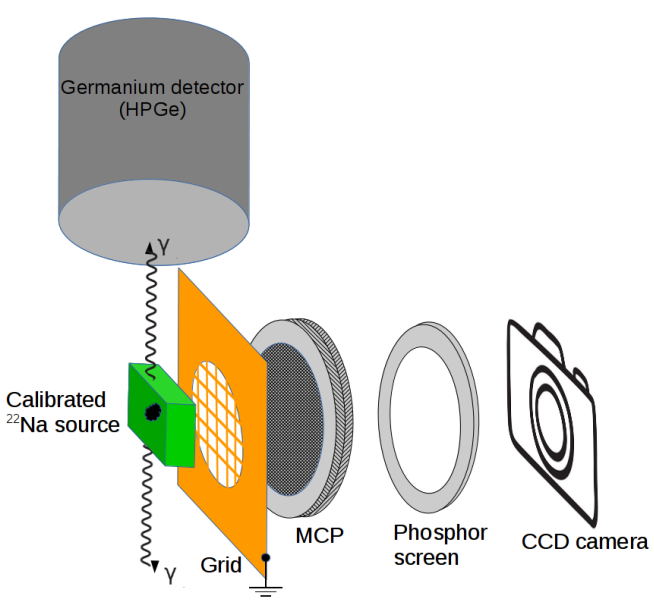

(b)

Figure 9. Schematic diagram of the setup used to estimate the positron beam intensity. (a): Positrons are implanted at $17 \mathrm{keV}$ into a PMMA support. (b): A calibrated ${ }^{22} \mathrm{Na}$ source located into a PMMA support. The annihilation $\gamma$-ray photons $(511 \mathrm{keV})$ were measured in both cases by means of a HPGe detector using the same geometry and attenuation effects. 
$17 \mathrm{keV})$ into the PMMA replica to minimize the positron reemission probability $(<0.1 \%)$ ensuring that positrons were annihilated within the PMMA. The shape of the hole helps to minimize the positron losses by backscattering $(<5 \%)[26]$.

The successive measurements of both PMMA supports of the calibrated source and of the replica with positrons implanted inside are performed with the same setup (see figure 9b) with the aim to reproduce the same geometrical effects for both measurements (distances, attenuation radiation, position of the source and detector, etc.). The source and the replica are centered in the same position at the center of the beam using sequentially an auxiliary laser that reproduces the trajectory of the positron beam and an auxiliary MCP behind the PMMA supports. This favors a direct comparison of the measurements and thus reduces the systematic sources of error.

To estimate the positron beam intensity three measurements of counts under the $511 \mathrm{keV}$ annihilation peak using the HPGe detector are performed: (i) background radiation of the laboratory, (ii) calibrated source (without beam) and (iii) positron beam implanted into the PMMA (without the calibrated source). The durations of these measurement are sufficient to yield a Poisson relative error of the count peak of the order or lower than $10^{-3}$.

By comparing the MCP count rate of the spots at $17 \mathrm{keV}$ with the effective beam intensity we can estimate an efficiency of $45 \pm 1 \%$ (stat.) $\pm 4 \%$ (syst.) at this energy. The error consists in the contribution of two terms, statistical and systematic. The latter is mainly given by the value declared by the manufacturer of the calibrated source (3\%) and by the indetermination of the backscattering effect of the positrons and the reproducibility of the position of both PMMA supports (about $0.3 \mathrm{~mm})$.

As mentioned in section 2, a setup configuration (see figure 1) is chosen to ensure that the positron intensity at the MCP is almost constant (within 1\%) for all incident energies. This setup includes a decelerator step at the end of the trajectory (see section 2). The effect of positron backscattering depends on the incident energy [26], but in this case it has a minimum or almost negligible influence at the MCP surface. Positrons that hit the surface of the tubes produce secondary electrons independently of the backscattering effect. For this reason, no correction of this effect is introduced.

After the determination of the spot number as a function of the kinetic energy the efficiency is normalized to the value obtained for $17 \mathrm{keV}$. Figure 10 shows the comparison of the efficiency obtained in the present work for positrons with the efficiency estimated for electrons [27, 28]. At low incident energies up to $1 \mathrm{keV}$ both efficiencies have the same trend with a maximum value around $70 \%$ at about 0.3 and $0.5 \mathrm{keV}$, respectively for electrons and positrons. This result demonstrates that MCPs are especially useful in experiments with particles, in this case positrons, at low energy. A similar trend, but with lower efficiency values, was obtained with low-energy trapped and bunched electrons and positrons (antiprotons also) in the range from 30 to $230 \mathrm{eV}$ [7]. The behavior of figure 10 for positrons and electrons follows to a first approximation the Bethe stopping-power formula that describes the energy loss into matter and the secondary "delta" electrons production as a function of the incident particles energy [29]. Secondary electron emission is favored at low incident energy (few hundred of $\mathrm{eV}$ ) when the atomic ionization at the surface of the MCP tubes is more efficient. The trends in figure 10 show that at higher incident energy the efficiency for positrons is higher with respect to that for electrons, for instance at $10 \mathrm{keV}$ it is about $28 \%$ for electrons while it reaches about $51 \%$ for positrons. Beyond the low energy coincidence $(<0.5 \mathrm{keV})$, 
the differences at higher energies may depend on the MCP configuration (open area, $L_{2}$ distance, etc.). The results could follow a similar trends using the same MCP configuration with particles and antiparticles. The efficiencies could be compared in a future work considering the possible effect of the gamma radiation coming from annihilation events when measuring positrons. This radiation promotes photoelectric and Compton electrons interacting with the surface of the MCP tubes and could presumably produce a measurable effect. We also remark that the efficiency of MCP detectors was determined for other ions and atoms such as in ref. [30].

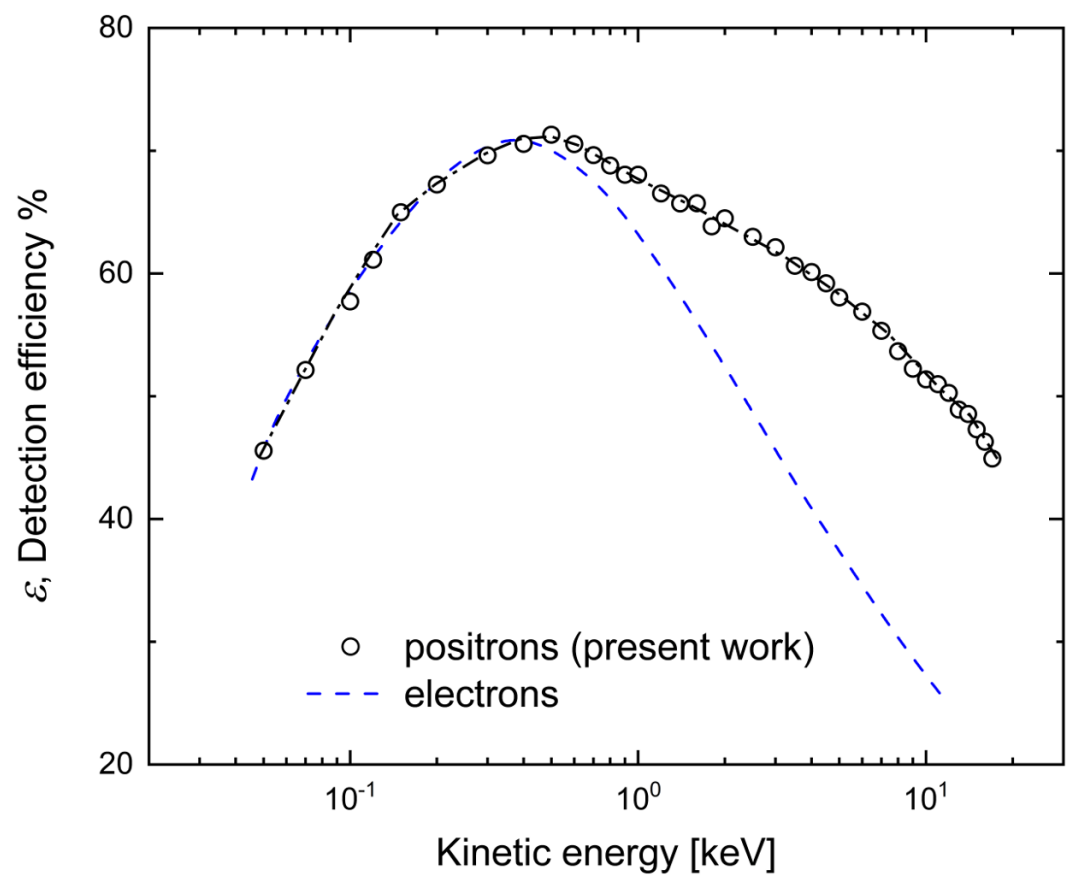

Figure 10. Efficiency as a function of incident energy of the positron beam. The contribution of statistical and systematic errors is of about $5 \%$ of the reported result. The black dashed line is only a visual guide. As a comparison, the typical efficiency estimated for electrons according to refs. [27, 28] is presented.

Nuclear emulsions were recently proved to be sensitive to low energy positrons with micrometric or sub-micrometric resolution [9, 25]. The MCP has clear advantages in terms of real-time monitoring, e.g. for interferometry applications, but it is not competitive yet with emulsions when high spatial resolution is required. On the other hand, the efficiency of nuclear emulsions at low energies is clearly less than that achieved by an MCP. Recent work reported in ref. [25] shows that for emulsion grains of $200 \mathrm{~nm}$ the efficiency approaches $5 \%$ for incident energies $\leqslant 1 \mathrm{keV}$. Instead, the efficiency of the emulsions increases as a function of the energy, reaching for instance about $80 \%$ at $10 \mathrm{keV} \mathrm{[25].}$

Taking into account the ideas presented in section 4 and in the present section it is possible to estimate the particle beam intensity as a function of the detection efficiency $\epsilon$ of the MCP (figure 10) and the overlapping probability $O v$ (figure 8 ). One obviously wants to assume an overlap probability 
as low as possible, with a minimum amount of data per frame. For instance, in this example we get 8.3\% overlap. The overlap factor is defined here as: $f_{O v}=(1-O v)^{-1}\left(f_{O v}=1.09\right.$ in this case). Thus the intensity of the beam $I_{\text {beam }}$ is computed as:

$$
I_{\text {beam }}=\epsilon^{-1} f_{O v} I_{\mathrm{MCP}}
$$

where $I_{\mathrm{MCP}}$ is the number of spots for unit of time read in the MCP. An example of an estimate for the data of figure 5 has been presented in section 3 . In this case, the beam intensity is about $2.9 \times 10^{3}$ positrons per second with an error of about $6 \%$, corresponding to 400 frames with 65 particles per frame on average (only $2.6 \times 10^{4}$ spots in total) obtained in $20 \mathrm{~s}$ of measuring time (plus the dead time between frames).

\section{Conclusions}

The present work contributes information regarding a digitizing method based on the detection of single particles. This offers a real-time diagnostic method to monitor and characterize a particle beam.

i) The spatial resolution of a single particle for this $\mathrm{MCP} /$ phosphor screen configuration is estimated in $60 \pm 2 \mu \mathrm{m}$.

ii) The detection efficiency of a microchannel plate (MCP) detector to low-energy positrons is studied in the energy range from $50 \mathrm{eV}$ to $17 \mathrm{keV}$. The measured efficiency varies between 45 and $71 \%$ depending on the positron incident energy.

iii) The method attributes the same statistical weight to each detected single particle, and thus it is possible to quantify the positron beam intensity and the geometric shape of the spot with limited statistics.

iv) We have estimated by means of simulations the trend of the overlapping effect as a function of the spot size of single particles, the overall width of the beam and the number of single spots for a given data frame.

The methodology presented in this work is applicable to the field of interferometry and deflectometry of antimatter. At the moment these techniques are considered a valid methodology to perform the first gravitational test with antimatter [15, 31]. As discussed in section 3, when the period of the patterns is smaller than the MCP resolution it becomes necessary to magnify the signal to use this real-time detector, for instance, using magnetic lenses (for charged particles) or using the moiré rotation effect. Therefore, it is potentially possible to distinguish interference or deflection patterns of antimatter using an MCP detector. In general, this method could be applied for antimatter particles, atoms and matter-antimatter hybrid atoms, such as positronium and muonium.

To improve the spatial resolution of this system an optimized MCP is required with smaller channels and a reduced distance to the phosphor screen $\left(\mathrm{L}_{2}\right)$. To reduce the acquisition time, especially in the case of intense positron beams, a phosphor screen is necessary with a shorter decay time. The P46 phosphor type seems suitable to this purpose, having a decay time five orders of magnitude less than the $\mathrm{P} 43$ phosphor used in this work. 


\section{Acknowledgments}

We would like to thank Martina Iannelli and Mauro Bombonati of Hamamatsu Photonics Italia for sending valuable information on the data and manufacturing characteristics of the MCP used in this work.

\section{References}

[1] H. Himura, S. Nakata and A. Sanpei, Applicability of micro-channel plate followed by phosphor screen to charged particles, Rev. Sci. Instrum. 87 (2016) 063306.

[2] R.C. Blase, R.R. Benke, G.P. Miller, K.S. Pickens and J.H. Waite, Microchannel plate detector detection efficiency to monoenergetic electrons between 3 and $28 \mathrm{keV}$, Rev. Sci. Instrum. 88 (2017) 053302 .

[3] K. Fehre, D. Trojanowskaja, J. Gatzke, M. Kunitski, F. Trinter, S. Zeller et al., Absolute ion detection efficiencies of microchannel plates and funnel microchannel plates for multi-coincidence detection, Rev. Sci. Instrum. 89 (2018) 045112.

[4] A. Tremsin, J. Vallerga and O. Siegmund, Overview of spatial and timing resolution of event counting detectors with microchannel plates, Nucl. Instrum. Meth. A 949 (2020) 162768.

[5] C. Amsler et al., $A \sim 100 \mu m$-resolution position-sensitive detector for slow positronium, Nucl. Instrum. Meth. B 457 (2019) 44.

[6] A.J. Peurrung and J. Fajans, A pulsed microchannel-plate-based non-neutral plasma imaging system, Rev. Sci. Instrum. 64 (1993) 52.

[7] G.B. Andresen, W. Bertsche, P.D. Bowe, C.C. Bray, E. Butler, C.L. Cesar et al., Antiproton, positron, and electron imaging with a microchannel plate/phosphor detector, Rev. Sci. Instrum. 80 (2009) 123701.

[8] A. Aghion et al., Nuclear emulsions for the detection of micrometric-scale fringe patterns: an application to positron interferometry, JINST 13 (2018) P05013.

[9] S. Aghion et al., Detection of low energy antimatter with emulsions, 2016 JINST 11 P06017 [arXiv: 1605.03944].

[10] S. Sala, A. Ariga, A. Ereditato, R. Ferragut, M. Giammarchi, M. Leone et al., First demonstration of antimatter wave interferometry, Sci. Adv. 5 (2019) eaav7610.

[11] A.Y. Barnyakov et al., Response of microchannel plates in ionization mode to single particles and electromagnetic showers, Nucl. Instrum. Meth. A 879 (2018) 6 [arXiv: 1707. 08503].

[12] S. Aghion et al., A moiré deflectometer for antimatter, Nature Commun. 5 (2014) 4538.

[13] J.L. Wiza, Microchannel plate detectors, Nucl. Instrum. Meth. 162 (1979) 587.

[14] K.A. Vereshchagin et al., On the decay time of luminescent screens of picosecond image tubes when measuring repeated signals in the accumulation mode, Bull. Lebedev Phys. Inst. 42 (2015) 7.

[15] A. Ariga et al., The QUPLAS experimental apparatus for antimatter interferometry, Nucl. Instrum. Meth. A 951 (2020) 163019.

[16] P.J. Schultz and K.G. Lynn, Interaction of positron beams with surfaces, thin films, and interfaces, Rev. Mod. Phys. 60 (1988) 701.

[17] N.E. Day, Estimating the components of a mixture of normal distributions, Biometrika 56 (1969) 463. 
[18] H. Sun and S. Wang, Measuring the component overlapping in the gaussian mixture model, Data Min. Knowl. Discov. 23 (2011) 479.

[19] M. Jones, M. Harvey, W. Bertsche, A.J. Murray and R.B. Appleby, Measuring the gain of a microchannel plate/phosphor assembly using a convolutional neural network, IEEE Trans. Nucl. Sci. 66 (2019) 2430.

[20] M. Dapor, A. Miotello and D. Zari, Monte carlo simulation of positron-stimulated secondary electron emission from solids, Phys. Rev. B 61 (2000) 5979.

[21] P.G. Merli, G.F. Missiroli and G. Pozzi, On the statistical aspect of electron interference phenomena, Am. J. Phys. 44 (1976) 306.

[22] A. Tonomura, J. Endo, T. Matsuda, T. Kawasaki and H. Ezawa, Demonstration of single-electron buildup of an interference pattern, Am. J. Phys. 57 (1989) 117.

[23] S.R. Müller, P. Nedelec and M.K. Oberthaler, From classical xenon fringes to hydrogen interferometry, New J. Phys. 22 (2020) 073060.

[24] G. Oster, M. Wasserman and C. Zwerling, Theoretical interpretation of moiré patterns, J. Opt. Soc. Am. 54 (1964) 169.

[25] L. Anzi, A. Ariga, A. Ereditato, R. Ferragut, M. Giammarchi, G. Maero et al., Sensitivity of emulsion detectors to low energy positrons, 2020 JINST 15 P03027.

[26] J. Makinen, S. Palko, J. Martikainen and P. Hautojarvi, Positron backscattering probabilities from solid surfaces at 2-30 keV, J. Phys. Cond. Mat. 4 (1992) L503.

[27] M. Galanti, R. Gott and J.F. Renaud, A high resolution, high sensitivity channel plate image intensifier for use in particle spectrographs, Rev. Sci. Instrum. 42 (1971) 1818.

[28] Hamamatsu MCP guide, MCP assembly, tmcp9002e01, Japan (2006) [https://studyres.com/doc/17571294/hamamatsu-mcp-guide].

[29] S.M. Seltzer and M.J. Berger, Evaluation of the collision stopping power of elements and compounds for electrons and positrons, Int. J. Appl. Radiat. Isot. 33 (1982) 1189.

[30] J. Oberheide, P. Wilhelms and M. Zimmer, New results on the absolute ion detection efficiencies of a microchannel plate, Meas. Sci. Technol. 8 (1997) 351.

[31] D. Krasnický et al., AEgIS experiment commissioning at CERN, AIP Conf. Proc. 1521 (2013) 144. 